\title{
Preventing and managing exacerbations in COPD - critical appraisal of the role of tiotropium
}

\author{
Donald P Tashkin \\ Department of Medicine, Division \\ of Pulmonary and Critical Care \\ Medicine, David Geffen School \\ of Medicine at UCLA, Los Angeles, \\ CA, USA
}

This article was published in the following Dove Press journal: International Journal of COPD

17 February 2010

Number of times this article has been viewed
Abstract: The course of COPD is punctuated by acute exacerbations that are associated with an increase in the morbidity and mortality related to this chronic disease and may contribute to its rate of progression. Therefore, preventing and treating exacerbations are major goals of COPD management. The role of tiotropium in the prevention of exacerbations has been investigated in several placebo-controlled randomized clinical trials varying in duration from 3 months to 4 years in patients with moderate to very severe COPD. In all of these trials, tiotropium has uniformly reduced the proportion of patients experiencing at least one exacerbation and delayed the time to the first exacerbation compared with placebo. In the longer trials ( $\geq 6$ months' duration) tiotropium has also reduced the exposure-adjusted incidence rate of exacerbations. In trials of at least 1 year in duration, tiotropium either significantly reduced the risk of hospitalization for an exacerbation and/or the proportion of patients with an exacerbation-related hospitalization. In a meta-analysis that included 15 trials of tiotropium vs either placebo $(n=13)$ and/or a longacting beta-agonist (LABA; $n=4$ ), tiotropium significantly reduced the odds of experiencing an exacerbation compared to placebo as well as a LABA. The potential additive benefits of tiotropium to those of a LABA and/or inhaled corticosteroid in reducing exacerbations require further investigation. The mechanism whereby tiotropium reduces exacerbations is not due to an anti-inflammatory effect but more likely relates to its property of causing a sustained increase in airway patency and reduction in hyperinflation, thereby counteracting the tendency for respiratory insults to worsen airflow obstruction and hyperinflation. For the management of acute exacerbations, an increase in short-acting inhaled bronchodilators is recommended as needed, while the potential role of long-acting bronchodilators, such as tiotropium, in conjunction with short-acting agents, is unclear and warrants further study.

Keywords: exacerbations, COPD, tiotropium, hospitalization, cardiovascular safety

\section{Introduction and background Definition}

The clinical course of COPD is punctuated by acute exacerbations that have been defined as "a sustained worsening of the patient's condition, from the stable state and beyond normal day-to-day variations, that is acute in onset and necessitates a change in regular medication in a patient with underlying COPD". ${ }^{1}$ Specifically excluded from the definition are other causes of worsening symptoms, such as pneumonia, congestive heart failure and pulmonary embolism. Another definition that has been used in clinical trials involving daily diaries requires a worsening of COPD symptoms for $\geq 2$ consecutive days based on a diary card review in which patients report a worsening of at least 2 major symptoms (dyspnea, sputum volume and/or sputum 
color) or the occurrence of at least one minor symptom (sore throat, coryzal symptoms, otherwise unexplained fever and/or worsening cough or wheeze), as previously defined by Anthonisen et al. ${ }^{2}$ The severity of an exacerbation has been operationally defined as mild if symptoms are self-managed by the patient in his/her own normal environment (eg, by an increase in the use of rescue bronchodilator use) without the use of antibiotics or oral corticosteroids, moderate if the patient seeks additional medical care and receives a prescription for an antibiotic and/or oral corticosteroids and severe if the exacerbation requires hospitalization because of rapid deterioration. Since it is generally not feasible to reliably ascertain a worsening of symptoms beyond normal day-to-day variation in the absence of daily diaries, exacerbations are commonly defined by more readily recalled events requiring health-care utilization ( $\mathrm{HCU}$ ) that result in a therapeutic intervention (usually treatment with antibiotics and/or oral corticosteroids). The duration of an exacerbation is highly variable and can last as long as 2 weeks, although some of the consequences of an exacerbation (such as worsening of airflow obstruction ${ }^{3}$ and further impairment in quality of life $\mathrm{e}^{4}$ ) may require weeks to months to return to baseline and may never return to the pre-exacerbation level if an exacerbation recurs.

\section{Etiology}

The cause of an acute exacerbation of COPD is most often infectious and related to a viral and/or bacterial infection. ${ }^{5}$ It is likely that the microbiome (ie, what organisms are present in the lungs) plays a critical role in predicting exacerbation rate. Sethi et al have shown an association between an exacerbation and isolation of a new strain of a bacterial pathogen, supporting the causative role of bacteria in exacerbations of COPD. ${ }^{6}$ Noninfectious causes include climate change, worsening air pollution and noncompliance with the usually prescribed maintenance medication regimen. Airway inflammation plays an important role in the transition from the stable state to an exacerbation, as indicated by a substantial increase in both neutrophils and eosinophils in induced sputum during an exacerbation compared to the stable condition. ${ }^{7}$ Perera et $\mathrm{al}^{8}$ have shown that persistence of increased airway inflammation during treatment of an acute exacerbation of COPD, as indicated by persistently high levels of interleukin (IL) IL-6 and IL-8 in sputum, predicted a prolonged recovery time. Evidence of increased systemic inflammation (indicated by a high serum C-reactive protein concentration) 2 weeks after an exacerbation has also been shown to be a predictor of recurrent exacerbations. ${ }^{8}$

\section{Frequency of exacerbations}

Exacerbations occur with increasing frequency in relation to the severity of COPD. For example, in the 3-year ISOLDE trial of inhaled corticosteroids (ICS) in COPD, ${ }^{9}$ the annual rate of HCU-defined exacerbations was 2.6, 1.7 and 1.2 in those patients with an $\mathrm{FEV}_{1}<1.25 \mathrm{~L}, 1.25$ to $1.54 \mathrm{~L}$ and $>1.54 \mathrm{~L}$, respectively. Using a symptom-based definition, Miravitilles et $\mathrm{al}^{10}$ reported a mean number of exacerbations per year of 2.3, 1.9 and 1.6 in patients with an $\mathrm{FEV}_{1}$ of $<40 \%, 40 \%$ to $59 \%$ and $\geq 60 \%$, respectively. In the 4-year UPLIFT trial of tiotropium vs placebo, the proportion of subjects with $\geq 1$ HCU-defined exacerbation per year was $80 \%, 72 \%$ and $60 \%$ in those with GOLD severity stage IV, III and II, respectively. ${ }^{11}$ In a 1-year prospective observational study of 351 patients with COPD seen in a primary care setting, those with mild to moderate disease had a mean number of exacerbations of 2.3, compared to 3.2 for patients with more severe disease. ${ }^{12}$ Thus, some patients with relatively mild disease may, like their more severe counterparts, also be predisposed to frequent exacerbations indicating increased susceptibility to these acute events. ${ }^{13}$ The most reliable predictor of a subsequent exacerbation in any given patient appears to be a history of previous exacerbations. ${ }^{13}$ It has been suggested that patients with frequent exacerbations (eg, $\geq 2$ per year) may represent a distinct phenotype of COPD, ${ }^{14}$ with implications for targeted preventative therapy.

\section{Impact of exacerbations}

Exacerbations have a number of untoward consequences both for the patient and society. First, an exacerbation of COPD has a negative impact on health-related quality of life (HRQoL), or health status, ${ }^{15}$ a greater annual frequency of exacerbations has been shown to be associated with higher scores on the St. George's Respiratory Questionnaire, in which higher scores represent worse health status. Similarly, Spencer et al have shown that the greater the frequency of exacerbations, the worse the annual rate of decline in health status. ${ }^{16}$ Following treatment of an exacerbation, HRQoL improves over several weeks (indicating a reciprocal worsening of health status in the run-up to an exacerbation), but up to 3 to 6 months may be required for health status to return to its pre-exacerbation level. Moreover, if another exacerbation occurs before an earlier one has completely resolved, health status may never return to baseline. ${ }^{16}$

Second, exacerbations are associated with a further impairment in lung function, contributing to the prominence of dyspnea as a major symptom. Since it is logistically difficult to track lung function prospectively in patients with 
COPD to document the worsening of lung function that occurs during the development of an exacerbation, a strategy that has been used to determine the degree of impairment in lung function accompanying an evolving exacerbation is to perform serial measurements of lung function during the recovery phase of a treated exacerbation since any improvement occurring during this phase must reflect reciprocal worsening that occurred during the run-up to an exacerbation. Stevenson et $\mathrm{al}^{3}$ measured airflow $\left(\mathrm{FEV}_{1}\right)$, airway resistance and inspiratory capacity (IC) for the first 3 days of a hospitalized exacerbation, at discharge and 42 days after admission. They found that lung function improved relatively little during the first 3 days of treatment but subsequently improved progressively up to 42 days along with a progressive reduction in dyspnea, the improvement in inspiratory capacity being more than twice the magnitude of that in $\mathrm{FEV}_{1}$ with relatively little change in airway resistance. These findings indicate that exacerbations are associated with greater worsening of hyperinflation than of airflow and are consistent with current concepts implicating hyperinflation as the major mechanism contributing to dyspnea during physical exertion. ${ }^{17}$ Similar findings were reported by Parker et al ${ }^{18}$ who documented significant improvement in IC and reciprocal decreases in hyperinflation (functional residual capacity [FRC]) and air-trapping (residual volume [RV]) over the 2 weeks of treatment of an acute exacerbation of moderate severity with parallel improvements in dyspnea. These findings also provide a clue as to the mechanism whereby longacting inhaled bronchodilator therapy (eg, with tiotropium) might help prevent exacerbations. Tiotropium produces a sustained reduction in hyperinflation ${ }^{19}$ and improvement in airflow, ${ }^{20}$ thus raising the threshold for the perception of symptoms of dyspnea during insults to the lung that tend to worsen hyperinflation and airflow.

Third, it has also been argued that a greater frequency of exacerbations is associated with a greater rate of disease progression as reflected by a larger annual rate of decline in $\mathrm{FEV}_{1},{ }^{21,22}$ although additional confirmation of this proposed association is required.

Fourth, acute exacerbations are associated with increased health-care utilization, increasing the cost of COPD both for the patient and society. Health care services for exacerbations account for $50 \%$ to $75 \%$ of all COPD costs. ${ }^{23}$ For patients who seek care as outpatients, the treatment failure rate varies from 13 to $33 \% .{ }^{24}$ Of patients who receive treatment in an emergency department (ED), the relapse rate is $22 \%$ to $32 \%$, often requiring a repeat ED visit. ${ }^{25}$ Approximately 721,000 hospitalizations for a COPD exacerbation were reported in $2005 .{ }^{26}$ For patients hospitalized for COPD, the in-hospital mortality overall is $\sim 2.5 \% .{ }^{27}$ However, for those requiring admission to an intensive care unit for hypercapnic ventilatory failure, the in-hospital mortality is $20 \%$ to $24 \%{ }^{28}$ In a 4-year prospective study of 205 patients hospitalized for COPD, 8.3\% died during their first admission, and 24\%, $33 \%, 39 \%$ and $49 \%$ died within 6 months, 1 year, 2 years and 4 years of admission, respectively. Thus, exacerbations are an important contributor to mortality in COPD. ${ }^{29}$

Because of the significant increase in morbidity and mortality from COPD exacerbations, the prevention and treatment of exacerbations are a major goal of COPD management according to current guidelines (GOLD) and a key outcome of interventional clinical trials. ${ }^{30}$

\section{The role of tiotropium}

Tiotropium has been found to be effective in preventing exacerbations, but its potential role in the management of an acute exacerbation requires further study. This article reviews the evidence supporting its role in reducing exacerbations and the limited available data concerning its use in treating an exacerbation.

\section{Preventing exacerbations}

Several double-blind, randomized controlled clinical trials of 3 months to 1 year in duration comparing tiotropium with placebo have evaluated the efficacy of tiotropium in preventing exacerbations of COPD as either a key secondary endpoint ${ }^{31-33}$ or as a primary endpoint. ${ }^{34,35}$ For most of these studies exacerbations were defined as a complex of respiratory events (cough, wheezing, dyspnea or sputum production) lasting $\geq 3$ days and generally requiring treatment with antibiotics and/or oral corticosteroids. Exacerbations were detected through careful monitoring of adverse events. Severe exacerbations were defined as those requiring hospitalization. Analyses were performed using the intention to treat principle for all patients remaining on-treatment. Several different endpoints for exacerbations were used in these trials to a varying degree. These are enumerated below, along with the method used for analyzing each of the endpoints.

1. Number of exacerbations treated with antibiotics and/or corticosteroids (ie, HCU-defined) per patient year. Between-group comparisons were made using Poisson regression with correction for treatment exposure and overdispersion.

2. Proportion of patients with $\geq 1$ HCU-defined exacerbation. Proportions between treatment groups were compared using Fisher's exact test. 
3. Time to first HCU-defined exacerbation. Treatment groups were compared using log-rank tests and the hazard ratio (HR) was estimated using Cox regression.

4. Number of exacerbation days per patient year. Poisson regression was used.

5. Number of COPD exacerbations leading to hospitalization per patient year. Poisson regression was used.

6. Proportion of patients with $\geq 1$ COPD exacerbation leading to hospitalization. Fisher's exact test was used for between-group comparisons.

7. The number of days hospitalized due to COPD exacerbations per year, using Poisson regression for analysis.

8. Time to the first COPD exacerbation leading to hospitalization with comparison between treatment groups using log-rank tests and the HR derived from Cox regression.

In a 1-year registration study (incorporating two identical clinical trials) of a total of 921 patients (mean baseline $\mathrm{FEV}_{1}$ $38.1 \%-39.1 \%$ pred; $40 \%-44 \%$ continued to use previously prescribed ICS), Casaburi et $\mathrm{al}^{31}$ showed that the proportion of patients experiencing $\geq 1$ exacerbations was lower in the tiotropium group (36\%) than the placebo group (42\%) (14\% reduction; $P<0.05)$. Significantly fewer exacerbations were observed in the tiotropium group ( 0.76 events/patient-year) compared to the placebo group (0.95 event/patient-year) ( $20 \%$ reduction; $P=0.045$ ), and a significant increase in the time to the first exacerbation was also noted in the tiotropium group relative to placebo $(P=0.011)$. Moreover, fewer hospitalizations associated with exacerbations occurred in the tiotropium group ( 0.086 events/patient-year) compared to the placebo group ( 0.161 events/patient-year), a $47 \%$ reduction $(P=0.019)$, and the proportion of patients hospitalized for an exacerbation was lower in the tiotropium group $(5.5 \%)$ than the placebo group $(9.4 \%, 41 \%$ reduction, $P<0.05)$.

In a 3-month study of 1639 patients (1236 tiotropium, 403 placebo) with a mean $\mathrm{FEV}_{1}$ of $45.4 \%$ predicted, a COPD exacerbation occurred in a significantly lower proportion of tiotropium patients (14.6\%) than placebo patients (19.9\%) (26\% reduction; $P=0.0151){ }^{32}$ The time to first exacerbation was also prolonged in the tiotropium compared to the placebo group $(P=0.0092)$.

Brusasco et $\mathrm{al}^{33}$ compared health outcomes, including exacerbations, in a 6-month double-blind, double-dummy study of 1207 COPD patients (mean baseline FEV $37.7 \%-39.2 \%$ pred) who received tiotropium $(\mathrm{n}=402)$, salmeterol $(n=405)$ or placebo $(n=400)$. Tiotropiumtreated patients had significantly fewer COPD exacerbations (1.07) and exacerbation days (17.2) per patient-year than those treated with placebo (1.49 and 25, respectively).
The reductions in exacerbations and exacerbations days per patient-yearr with tiotropium compared to placebo were $28 \%$ and $31 \%$, respectively ( $P=0.025$ for both). Tiotropium also significantly delayed the time to the first COPD exacerbation compared with placebo $(P<0.01)$. While tiotropium-treated patients had fewer hospital admissions for an exacerbation than placebo-treated patients, the difference was not statistically significant, nor did the time to the first hospital admission for a COPD exacerbation differ between the tiotropium and placebo groups, possibly due to the relatively small number of exacerbation-related hospitalizations during this 6-month study. Interestingly, however, significantly fewer all-cause hospital admissions occurred in the tiotropium than the placebo group ( $0.43 \mathrm{vs}$ 0.86 , respectively; $P<0.05$ ). No significant differences were noted between the tiotropium and salmeterol treatment groups for any exacerbation variable.

The effect of tiotropium vs placebo on exacerbations was the primary aim of a multi-center, 1-year French study (MISTRAL) $^{34}$ that included 1010 subjects with a baseline $\mathrm{FEV}_{1}$ of $47.6 \%$ to $48.2 \%$ predicted. Continued use of ICS (reported at baseline by $61.6 \%-65.0 \%$ of the subjects) was permitted throughout the trial. For this study, an exacerbation was defined as the onset of $\geq 1$ symptom (worsening dyspnea, cough or sputum; sputum purulence; fever) lasting $\geq 2$ days and requiring a new prescription or an increase in the dose of $\beta_{2}$-agonists, antibiotics, corticosteroids or bronchodilators. The severity of an exacerbation was defined as follows: mild - one or two of the above symptoms; moderate $-\geq 3$ of the above symptoms excluding a severe exacerbation; severe - exacerbation requiring hospitalization or meeting at least one criterion based on pre-defined drops in $\mathrm{FEV}_{1}$ and/or peak expiratory flow or defined changes in $\mathrm{PaO}_{2}$ and/or $\mathrm{PaCO}_{2}$. For comparison with the results of other studies, a more generalized HCU-based definition of exacerbation severity was used: severe - requiring hospitalization; moderate - requiring treatment with systemic steroids and/or antibiotics; mild - all remaining exacerbations. The proportion of patients experiencing $\geq 1$ exacerbations during the 1-year treatment period was significantly lower in the tiotropium than the placebo group $(17 \%$ reduction; $P<0.01)$. Moreover, tiotropium-treated patients had significantly fewer COPD exacerbations ( $35 \%$ reduction; $P<0.001)$ and exacerbation days (37\% reduction; $P<0.001)$ than placebo-treated patients, and tiotropium significantly delayed the time to the first exacerbations by $\sim 100$ days compared with placebo $(P<0.001)$. Using the generalized definition of exacerbation severity, tiotropium reduced the proportion of patients 
experiencing $\geq 1$ moderate to severe exacerbation by $20 \%$ $(P<0.001)$ and reduced the number of moderate to severe exacerbations by $35 \%(P<0.001)$ compared to placebo. While tiotropium resulted in numerically fewer exacerbation-related hospitalizations and hospital days, the differences from placebo were not statistically significant. Tiotropium significantly reduced the number of exacerbations compared with placebo, irrespective of COPD severity (ie, $\mathrm{FEV}_{1}>$ or $\leq 50 \%$ predicted). Although tiotropium numerically reduced exacerbations irrespective of concomitant ICS use or exacerbation history during the year preceding the trial, this reduction failed to reach statistical significance in those not receiving concomitant ICS or those with infrequent exacerbations in the preceding year.

A 6-month placebo-controlled trial involving 26 Veterans Affairs medical centers in the US was conducted to evaluate the efficacy of tiotropium in preventing exacerbations of COPD as the primary outcome. ${ }^{35} \mathrm{~A}$ unique feature of this study was the permissive use during the entire treatment period of all prescribed concomitant respiratory medication (including ICS and long-acting beta-agonists [LABAs]), other than anticholinergic medication, in addition to the study drug, thus mimicking "real-world" conditions. Another unique feature of this study was its ability to track lower respiratory serious adverse events (SAEs), essentially capturing exacerbation-related hospitalizations, using electronic medical records from the VA system even in patients who prematurely withdrew from the trial. ${ }^{36}$ Randomized participants included 1829 patients with moderate to severe COPD (mean baseline $\mathrm{FEV}_{1} 36 \%$ predicted), $98 \%-99 \%$ of whom were male. Tiotropium significantly reduced the percentage of patients experiencing $\geq 1$ exacerbation during the 6-month trial compared to placebo (27.9\% vs $32.3 \%$, respectively; $P=0.037$ ). Tiotropium also reduced the percentage of patients with $\geq 1$ exacerbation-related hospitalization compared to placebo (7.0 vs 9.5\%), although this difference just missed statistical significance $(P=0.056)$. The analysis assumed that the 47 tiotropium patients (5.1\%) and 66 placebo patients $(7.2 \%)$ who withdrew from the study before an event had no event. However, when incidence rates of serious (hospitalized) exacerbations per 100 patient-years were calculated for those subjects who prematurely discontinued the trial, these rates were $\sim 2$-fold higher in the placebo group during the remainder of the protocol-defined trial period after premature cessation of the study drug (22.1) than during the actual treatment period prior to premature withdrawal from the trial (12.4). In contrast, the rates were similar for these two periods in the tiotropium group (9.2 vs 8.8 , respectively). ${ }^{36}$ Since more placebo than tiotropium patients withdrew prematurely from the treatment phase of the study, these findings imply a bias in favor of placebo if the analysis included only events that occurred during actual treatment with the study drug.

Analysis of secondary outcomes from the VA trial showed that tiotropium prolonged the time to the first exacerbation (HR 0.83 [95\% confidence interval, CI, 0.70-0.98]; $P=0.028)$, as well as to the first exacerbation-related hospitalization (HR 0.73 [CI, 0.53-1.01]; $P=0.055$ ), although the latter finding was of borderline statistical significance. Compared to placebo, tiotropium also reduced the overall frequency of exacerbations by $19 \%(P=0.031)$ and of hospitalizations due to exacerbations by $28 \%(P=0.047)$ and decreased the number of exacerbation days by $21 \%(P=0.019)$. In subgroup analyses of the VA trial data, tiotropium nearly uniformly reduced exacerbations for all subsets included in the analysis: age ( $>70,61-70$ and $<61$ years), current smoking, FEV ( $>49 \%, 35 \%-49 \%$ and $<35 \%$ predicted), hospitalization in the past year, antibiotic use in the past year, home oxygen use, ICS use, LABA use and theophylline use (Figure 1). These results imply a benefit of tiotropium in reducing exacerbations when used in conjunction with other prescribed long-acting inhaled respiratory medication.

In addition to the aforementioned study of Brusasco et al, ${ }^{33}$ which compared the impact of tiotropium with that of salmeterol (as well as placebo) on exacerbations, two additional reports compared the effect of tiotropium on exacerbations with that of another active comparator, ipratropium ${ }^{37}$ or salmeterol. ${ }^{38}$ The results of two identical multi-center 1-year trials in Belgium and the Netherlands of tiotropium once daily $(\mathrm{n}=356)$ vs ipratropium four times daily $(\mathrm{n}=179)$ in patients with a mean $\mathrm{FEV}_{1}$ of $39.4 \%$ to $41.9 \%$ predicted were combined into a single report. ${ }^{37}$ Over $80 \%$ of the patients continued to use prescribed ICS during the trial. The proportion of patients with $\geq 1$ exacerbations during the 1-year treatment period was significantly lower in the tiotropium group (35\%) than the ipratropium group $(46 \%)(P=0.014)$. The number of exacerbations per patient-year was 0.73 in the tiotropium group and 0.96 in the ipratropium group $(24 \%$ reduction with tiotropium, $P=0.006$ ). Furthermore, the number of exacerbation days/patient-year was 39\% lower in the tiotropium group (10.8 vs 17.7 in the ipratropium group; $P=0.002$ ). The time to first exacerbation was significantly longer in the tiotropiumtreated patients $(P=0.008)$. Similar findings were noted for hospitalized exacerbations, although most of these failed to reach statistical significance. The proportion of patients with an exacerbation-related hospitalization was $7.3 \%$ and $11.7 \%$ in the tiotropium and ipratropium groups, respectively $(P=0.11)$. 


\section{Characteristic}

Overall $(n=1829)$

Age

$>70$ y $(n=756)$

$61-70$ y $(n=673)$

$<61$ y $(n=400)$

Current smoker

No $(n=1294)$

Yes $(n=535)$

$\mathrm{FEV}_{1}$

$>49 \%$ predicted $(n=287)$

$35 \%-49 \%$ predicted $(n=610)$

$<35 \%$ predicted $(n=931)$

Hospitalization in the past year

No $(n=1543)$

Yes $(n=286)$

Prednisone use in the past year No $(n=1334)$

Yes $(n=492)$

Antibiotic use in the past year

No $(n=1001)$

Yes $(n=826)$

Home oxygen use

No $(n=1298)$

Yes $(n=531)$

Inhaled steroid use

No $(n=787)$

Yes $(n=1041)$

Long-acting $\beta$-agonist use

No $(n=1115)$

Yes $(n=713)$

Theophylline use

No $(n=1564)$

Yes $(n=264)$
Odds Ratio for $\geq 1$ COPD Exacerbation

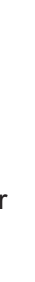

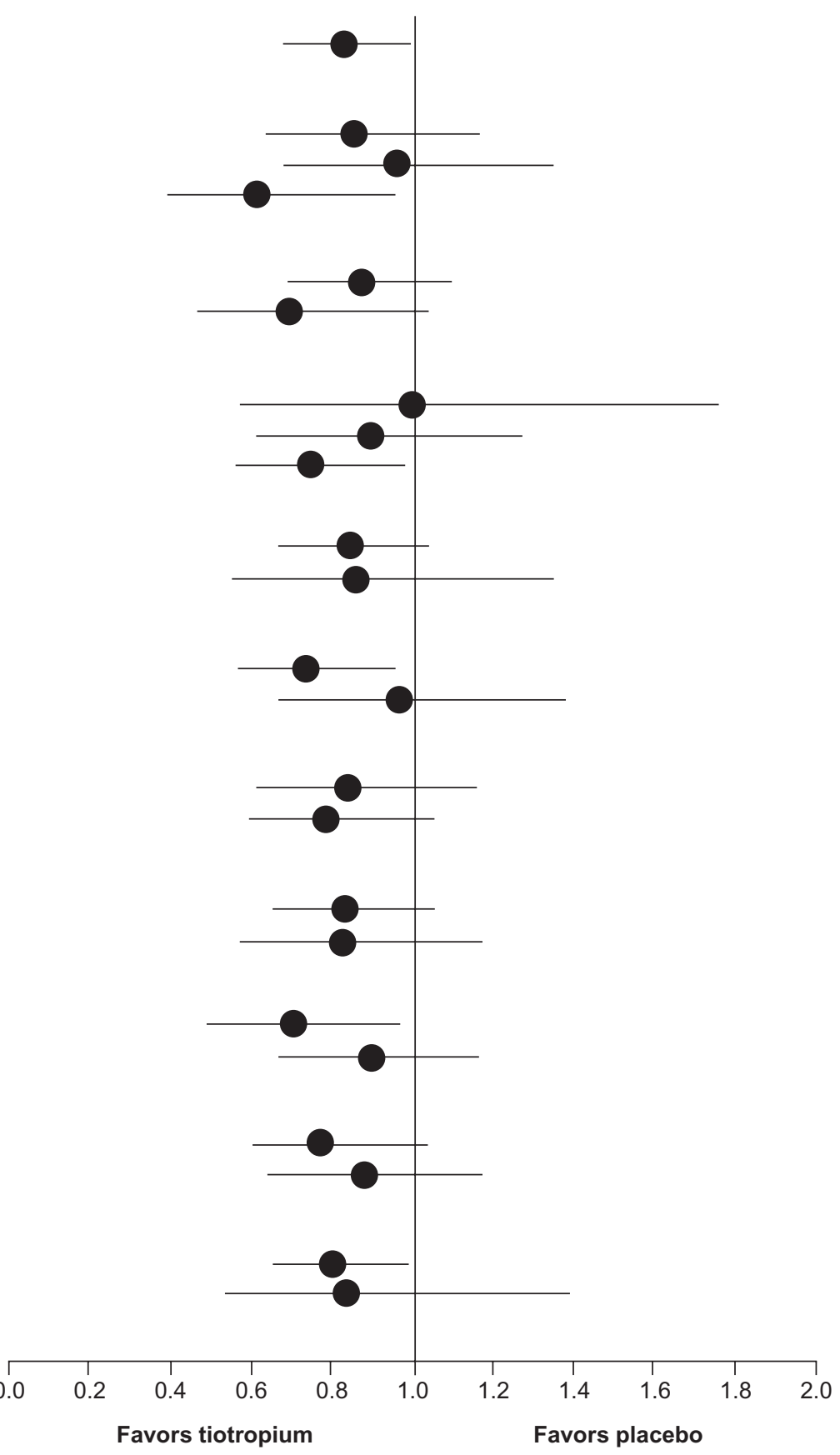

Figure I Odds ratios and $95 \%$ confidence intervals ( $\mathrm{Cls}$ ) for reduction in first COPD exacerbation with tiotropium according to selected baseline characteristics. Numbers in parentheses represent sample size for that subset. Error bars represent $95 \%$ Cls. Reproduced with permission from Niewoehner DE, Rice K, Cote C, et al. Prevention of exacerbations of chronic obstructive pulmonary disease with tiotropium, a once-daily inhaled anticholinergic bronchodilator. Ann Intern Med. 2005; I43:3I 7-26. ${ }^{35}$ Copyright $(C)$ 2005 American College of Physicians.

The number of hospitalizations per patient-year was 39\% lower in the tiotropium group ( 0.10 vs $0.16, P=0.08)$. Furthermore, the time to first hospitalized exacerbation was significantly longer in the tiotropium-treated patients $(P=0.048)$. These findings are all the more remarkable since ipratropium itself has been shown to reduce exacerbation frequency significantly compared with regularly scheduled albuterol alone. ${ }^{39}$
Another study compared the efficacy of tiotropium once daily with that of salmeterol $50 \mathrm{mcg}$ twice daily over 12 weeks in 563 patients with a mean $\mathrm{FEV}_{1}$ of $37.7 \%$ predicted. ${ }^{38}$ In this short-term study the overall incidence of COPD exacerbations was low and the percentage of patients with $\geq 1$ exacerbation was fairly comparable between the tiotropium and salmeterol groups ( 9 vs $11 \%$, respectively, $P=0.37$ ), as 
was the frequency of exacerbations, exacerbation days and time to first exacerbation. Non-significantly fewer tiotropiumthan salmeterol-treated patients experienced an exacerbation-related hospitalization ( $4 \mathrm{vs} 9$, respectively; $P=0.16$ ). Thus, the results of this study were consistent with those of the 6-month study of Brusasco et $\mathrm{al}^{33}$ with respect to the lack of significant differences between tiotropium and salmeterol in preventing exacerbations, although the relatively short duration of these trials may have reduced the ability to demonstrate possible differences between the two treatments in their effects on exacerbations.

The effects of tiotropium in preventing HCU-defined exacerbations (requiring treatment with oral corticosteroids and/or antibiotics or hospitalization) were also compared with those of the salmeterol/fluticasone combination (SFC) in a 2-year study (INSPIRE) of 1323 patients with severe/very severe COPD (mean baseline FEV $139.4 \%$ predicted). ${ }^{40}$ The primary endpoint was the mean annual number of HCU-defined exacerbations. The annual exacerbation rate was essentially the same in the tiotropium and SFC groups: 1.32 and 1.28, respectively; incidence rate ratio 0.97 [95\% CI, 0.84-1.12]; $P=0.66$ ). On the other hand, the nature of the exacerbations differed between the two groups in that a significantly lower rate of exacerbations treated with oral corticosteroids was noted in the SFC than the tiotropium group ( 0.69 vs 0.85 , respectively; rate ratio 0.81 [95\% CI, 0.67-0.99]; $P=0.039$ ), while a significantly higher rate of exacerbations treated with antibiotics was observed in the SFC than the tiotropium group (0.97 vs 0.82 , respectively; rate ratio 1.19 [95\% CI, 1.02-1.38]; $P=0.028)$.

The higher rate of exacerbations requiring oral corticosteroids in the tiotropium group might possibly reflect the need for an anti-inflammatory agent by some of these patients for protection against exacerbations. This could have been particularly true for the $\sim 50 \%$ of the tiotropium subjects who had been receiving ICS prior to the trial, since withdrawal of ICS in these subjects at trial entry could have predisposed them to an exacerbation requiring oral corticosteroids after the potentially beneficial effects of the initial two-week course of oral prednisolone (which all patients in the trial received during a 2-week run-in period of intensified therapy) wore off. An increased risk of a COPD exacerbation after withdrawal of ICS has been reported previously. ${ }^{41,42}$

On the other hand, the higher rate of exacerbations requiring antibiotics in the SFC group might have been due to fluticasone-induced impairment of local immune resistance to lower respiratory infection since the incidence of pneumonia with SFC was twice as high as that with tiotopium (8 vs 4\%, respectively; HR 1.94 [95\% CI, 1.19-3.17]; $P=0.008$ ). The latter findings are also consistent with the an increase in pneumonia incidence in the fluticasone-containing arms of the TORCH trial, ${ }^{43}$ an increased risk of pneumonia with ICS in a recent meta-analysis ${ }^{44}$ and an increased risk of pneumonia hospitalizations with ICS in an observational study. ${ }^{45}$ In contrast, tiotropium has not been found to be associated with an increased incidence of pneumonia. ${ }^{20}$

The two co-primary endpoints of the recent 4-year Understanding Potential Long-Term Impacts on Function with Tiotropium (UPLIFT) trial of tiotropium vs placebo were the annual rates of change in pre- and post-bronchodilator $\mathrm{FEV}_{1}$ over 4 years, testing the hypothesis that tiotropium might influence the natural progression of COPD. ${ }^{20}$ Nearly 6000 patients (2896 on tiotropium and 3006 on placebo) with moderate, severe or very severe COPD (45\%-46\%, 44\% and $8 \%-9 \%$, respectively), including $\sim 25 \%$ females, were studied. The impact of tiotropium vs placebo on exacerbations and related hospitalizations was a pre-specified, key secondary endpoint. A unique feature of this global study involving 490 investigational centers in 37 countries was the permissive use of all concomitant prescribed respiratory medication, in addition to the study drug, over the course of the trial, with the exception of inhaled anticholinergics. Tiotropium resulted in a significant delay in the time to the first exacerbation compared to placebo with a reduction in the risk of exacerbations by $14 \%$ (HR $0.86[95 \%$ CI, 0.81-0.91]; $P<0.001$ ). Tiotropium also significantly reduced the frequency of exposure-adjusted exacerbations (number per patient-year) $(0.73 \pm 0.02)$ compared to placebo $(0.85 \pm 0.02)$ with a relative risk of 0.86 [95\% CI, 0.81-0.91; $P<0.001]$. In addition, tiotropium reduced the risk of exacerbation-related hospitalization by $14 \%$ (HR $0.86[95 \%$ CI, 0.78-0.95]; $P=0.002$ ). Complementing the latter benefit was the finding that tiotropium reduced the incidence (per 100 patient-years) of serious lower respiratory adverse events, including the incidence of respiratory failure.

In a sub-group analysis of the 2739 GOLD stage II subjects (mean $\mathrm{FEV}_{1} 59 \%$ predicted) within the larger total UPLIFT trial population, time to first exacerbation and time to first exacerbation resulting in hospital admission were significantly longer in the tiotropium than the placebo group (HR 0.82 [95\% CI, 0.75-0.90] and 0.74 [95\% CI, 0.62-0.88], respectively). ${ }^{11}$ These findings indicated at least comparable benefits of tiotropium in reducing exacerbations in patients with moderate COPD compared to those with severe disease, despite the fact that the former subjects had 
fewer exacerbations than the latter in both treatment groups. Another subgroup analysis compared the impact of tiotropium on exacerbations in relation to differing smoking status during the course of the trial, defined as continuing smoking, continuing ex-smoking and intermittent smoking. ${ }^{46}$ The rate ratios (RR; tiotropium/placebo) for the number of exacerbations per patient-year were roughly comparable across these different patterns of smoking during the study, ranging from 0.83 to 0.90 , although a significant reduction in exacerbations with tiotropium was observed only in the continuing ex-smokers (RR 0.83 [95\% CI, 0.77-0.90]), who comprised the largest proportion of the study population $(\sim 60 \%)$.

While a formal subgroup analysis of the UPLIFT data by concomitant LABA and/or ICS use has not yet been completed, it appears that the benefits of tiotropium on most outcomes, including reduction in exacerbations, were achieved irrespective of concurrent use of other prescribed long-acting inhaled respiratory medication (which was reported by over $60 \%$ of the participants at baseline and over $70 \%$ over the course of the trial). If this result is confirmed, the implications are that tiotropium may add to the benefit of existing LABA and/or ICS use in reducing exacerbations since the latter medications when used as monotherapy or in combination have previously been shown to be effective in preventing exacerbations. ${ }^{43}$

On the other hand, the results of the Canadian Optimal Treatment of COPD trial ${ }^{47}$ failed to show a significant additive effect of salmeterol or of the SFC combination when administered in addition to tiotropium on HCU-defined exacerbations (the primary endpoint). This study was a 1-year multi-center, randomized, double-blind, placebocontrolled trial of tiotropium once daily alone compared with the addition of either salmeterol $(50 \mu \mathrm{g})$ twice daily or the SFC (500 $\mu \mathrm{g}$ fluticasone $/ 50 \mu \mathrm{g}$ salmeterol) twice daily to tiotropium once daily in 449 patients with moderate to severe COPD (mean baseline post-bronchodilator $\mathrm{FEV}_{1}$ $41.2 \%-42.2 \%$ predicted) with a history of $\geq 1$ exacerbation of COPD in the previous year. Over the 1-year treatment period, the "triple" combination (tiotropium plus SFC) did not result in any significant difference from tiotropium alone in the proportion of patients with $\geq 1$ COPD exacerbation (60\% vs $62.8 \%$, respectively) or a significant reduction in the risk of an exacerbation compared to tiotropium alone (HR 0.80 [95\% CI, 0.60-1.08); $P=0.15$ ). On the other hand, compared to tiotropium alone, the "triple" combination did result in a significantly reduced incidence rate ratio for severe exacerbations, defined as exacerbation-related hospitalizations (0.53 [95\% CI, 0.33-0.86]). This study may have been underpowered to detect a significant difference in the primary endpoint. Moreover, salmeterol appears to be a less effective LABA than formoterol, as suggested by the failure of the salmeterol/tiotropium combination in this Canadian study to produce a significantly greater trough $\mathrm{FEV}_{1}$ after 1 year of therapy than tiotropium alone, in contrast to findings from several studies that the combination of tiotropium and formoterol produces additive bronchodilation over tiotropium alone, including additive effects in reducing hyperinflation, as recently summarized by Cazzola and Tashkin. ${ }^{48}$ It is possible, therefore, that the combination of tiotropium with either formoterol alone or formoterol plus an ICS could result in additive benefits with respect to preventing exacerbations. Additional well-designed and adequately powered studies are required to explore this possibility.

\section{Meta-analysis}

A recent meta-analysis of randomized controlled trials, including 15 trials of tiotropium vs placebo and/or an active comparator (13 vs placebo; 3 vs salmeterol and 1 vs formoterol) examined the impact of tiotopium in reducing exacerbations. ${ }^{49}$ The results indicated a significantly reduced odds of experiencing an exacerbation with tiotropium vs placebo (odds ratio [OR] 0.69 [95\% CI, 0.61-0.78]), as well as with tiotropium vs LABA (OR 0.82 [95\% CI, 0.72-0.93]). Using a mixed treatment comparison meta-analysis, the authors also found a significantly reduced odds of experiencing an exacerbation with tiotropium vs ICS (OR 0.81 [95\% CI, 0.69-0.94]) and a nonsignificant reduction in the odds ratio for tiotropium vs ICS/LABA combination (OR 0.91 [95\% CI, 0.78-1.04]).

\section{Putative mechanism of exacerbation reduction with tiotropium}

Since most exacerbations are considered to have an infectious etiology ${ }^{5}$ and are associated with an increase in airway inflammation, ${ }^{7,8}$ the mechanism whereby a long-acting bronchodilator such as tiotropium might reduce or prevent exacerbations is unclear. Although tiotropium has been shown to have anti-inflammatory properties in cell-based studies, ${ }^{50,51}$ the clinical significance of these has not been demonstrated. To investigate the possibility that tiotropium might reduce exacerbations in part through an anti-inflammatory mechanism, Powrie et al ${ }^{52}$ studied the effect of a 1-year treatment with tiotropium vs placebo in 142 patients with COPD (mean $\mathrm{FEV}_{1} \sim 50 \%$ predicted) on sputum and serum inflammatory markers (sputum IL-6, IL-8 and MPO and serum IL-6 and CRP) at the same time that they examined the effect 
on exacerbations. Tiotropium resulted in a $52 \%$ reduction in the rate of exacerbations per patient year compared to placebo $(1.17 \pm 2.25$ [SD] vs $2.46 \pm 3.82$, respectively; $P=0.007)$ and a significant delay in the time (in days) to the first exacerbation (236 \pm 143 vs $157 \pm 124$ days, respectively; $P=0.009$ ). However, no differences in sputum or serum inflammatory markers were noted between tiotropium and placebo over the year-long study. The authors concluded that the reduction in exacerbations with tiotropium is unlikely to be mediated by a reduction in airway or systemic inflammation.

One possible mechanism whereby tiotropium might reduce exacerbations may be through improved clearance of respiratory secretions resulting from the bronchodilatorinduced increase in airway patency. ${ }^{53}$ Another possible mechanism may be mediated through the sustained improvement in airflow and reduction in hyperinflation due to its long-acting bronchodilator properties ${ }^{20,54}$ that result in a reduction in dyspnea. ${ }^{31,19,55}$ Exacerbations are associated with worsening lung function, ${ }^{3,18}$ including an increase in hyperinflation, that results in increasing dyspnea that leads the patient to seek medical attention, thus fulfilling a HCU-defined exacerbation. It is possible, therefore, that the sustained improvement in lung function achieved with a 24-hour-acting bronchodilator might raise the threshold for the perception of symptoms in response to various insults (including a respiratory tract infection) that might otherwise cause a critically severe impairment in lung function to a degree that produces symptoms that the patient deems warrants medical attention.

\section{Potential limitations}

Clinical trials that evaluate the impact of a therapeutic intervention on exacerbations, including the ones included in this review, are subject to a number of potential limitations. ${ }^{56,57}$ First, exacerbations are defined in different ways and there may be uncertainty as to the true occurrence of an exacerbation in accord with the pre-specified definition of an exacerbation (whether symptom- or event-based) that is ideally resolved using an independent, blinded adjudication process. Unfortunately, the latter process is rarely implemented. Second, it may be difficult, in some cases, to distinguish between a new exacerbation and one that is slow to resolve or has relapsed. This limitation would affect analyses of the frequency or rate of exacerbations but not a "survival curve" analysis of the time to the occurrence of the first exacerbation. Third, in most clinical trials there is no attempt to capture exacerbations after the patient prematurely withdraws from the trial. (The VA cooperative trial ${ }^{35,36}$ and
Canadian Optimal Treatment trial ${ }^{47,56}$ are rare exceptions). This creates a potential bias since exacerbation rates are much higher after premature discontinuation of study drug. ${ }^{36,57}$ This bias can only partially be adjusted for by a weighted statistical approach that takes into account the time spent in the trial, a method used in most of the trials reviewed herein. Fourth, variations in the effect of treatment between subjects assigned to different arms of a parallel-group study leads to overdispersion of residuals in the usual parametric analysis, causing inappropriate narrowing of the confidence intervals; this can be corrected by using a Poisson distribution with adjustment for overdispersion or by using a negative binomial error distribution. ${ }^{58,59}$ Such techniques were applied to the analysis of the exacerbation data in most of the tiotropium trials included in this review.

\section{Managing exacerbations}

According to current COPD guidelines, ${ }^{30}$ long-acting inhaled bronchodilators, such as tiotropium, have no defined role in the management of acute exacerbations. The home management of exacerbations involves increasing the dose and/or frequency of existing short-acting bronchodilators, preferably with a $\beta_{2}$-agonist, although, despite the absence of compelling evidence of the additional benefit of combinations of short-acting bronchodilators, it is suggested that an anticholinergic bronchodilator can be added until symptoms improve. Systemic corticosteroids are also recommended for 7 to 10 days in addition to bronchodilators because of evidence that they shorten recovery time, improve lung function and hypoxemia and reduced the risk of early relapse or treatment failure. In addition, antibiotics are recommended for patients with increased sputum purulence in addition to increased dyspnea and/or increased sputum volume and for patients with severe exacerbations requiring either invasive or noninvasive mechanical ventilation. For patients hospitalized for a COPD exacerbation, supplemental oxygen therapy is recommended, along with bronchodilators, systemic corticosteroids and, under the circumstances noted above, antibiotics. Patients not responding favorably to these measures may require mechanical ventilatory assistance, either noninvasive (preferred) or with intubation.

Regarding the choice of bronchodilators in either the home or the hospital setting, short-acting inhaled $\beta_{2}$-agonists are usually preferred, but, if a prompt response does not occur, the addition of a short-acting anticholinergic (ipratropium) is recommended. Unfortunately, few studies have evaluated the use of long-acting inhaled bronchodilators (either LABAs or tiotropium) during an exacerbation. 
Di Marco et $\mathrm{al}^{60}$ examined the effect of tiotropium once daily, formoterol twice daily and their combination administered using a 3-way crossover design with a random sequence to 21 patients (mean $\mathrm{FEV}_{1} 38 \% \pm 14 \%$ predicted) during an acute exacerbation of COPD managed outside the hospital. Lung function was measured serially over 24 hours during each medication sequence. Mean $\mathrm{FEV}_{1}, \mathrm{FVC}$ and $\mathrm{IC} \mathrm{AUC}_{0-12 \mathrm{~h}}$ and $\mathrm{AUC}_{0-24 \mathrm{~h}}$ after the combination of tiotropium and formoterol were significantly higher compared to either drug alone, while no significant differences were noted between tiotropium and formoterol monotherapy. Interestingly, the bronchodilating effects of each regimen were shorter in the setting of an acute exacerbation than has been generally observed in patients with stable COPD and, by 24 hours, had disappeared with the exception of a significant residual improvement in $\mathrm{FEV}_{1}$ with the combination only. This curtailment in the duration of action of these long-acting bronchodilators during an exacerbation may be due to several factors. One factor may be less effective delivery of the inhaled medication to the lower respiratory tract during an exacerbation due to the associated increase in airways obstruction from increased inflammation, airway mucus and bronchospasm. Another factor may involve functional antagonism, in which constrictor stimuli may be enhanced during an exacerbation, possibly related to the increase in inflammation, thereby requiring a greater dose and/or duration of bronchodilators to counteract the enhanced bronchoconstrictor tendency. The clinical implications of these findings regarding the bronchodilator management of acute exacerbations are that regularly scheduled long-acting inhaled bronchodilators and, in particular, the combination of tiotropium once daily and a LABA twice daily, have potential benefits for the management of an acute exacerbation, although additional treatment with short-acting bronchodilators is most likely required, together with the longer-acting agents, because of the shortened duration of action of the latter during an exacerbation compared to the stable state.

Drescher et $\mathrm{l}^{61}$ recently published the results of a singlecenter study concerning the possible benefits of incorporating long-acting inhaled bronchodilators (specifically tiotropium once daily and formoterol twice daily) into a respiratorytherapist directed program for the hospital management of severe COPD exacerbations in nonventilated patients. Since the authors had previously incorporated twice-daily treatment with formoterol into its inpatient program for managing COPD exacerbations, ${ }^{62}$ the addition of tiotropium to this preexisting program resulted in a new program of combination long-acting inhaled bronchodilator therapy. In addition to this combination of long-acting inhaled bronchodilators, patients received treatments with short-acting agents $\left(\beta_{2}\right.$-agonist and/or anticholinergic) as needed. The primary outcome of this retrospectively analyzed study was the total number of as-needed short-acting bronchodilator treatments per patient per admission following the implementation of the new inpatient bronchodilator program in comparison with similar data collected during a previous historical period when formoterol alone was routinely administered twice daily. Therefore, the differences between the two periods reflected primarily the novel addition of tiotropium to the pre-existing bronchodilator regimen. The authors found that the addition of tiotropium resulted in a significant reduction in the number of short-acting bronchodilator treatments per admission, as well as a significant reduction in the length of hospital stay of $\sim 1$ day, compared to the historical control group treated during the same season of the year (winter months); these benefits translated into a pharmacoeconomic advantage. The authors also found that tiotropium use in the in-hospital setting was well-tolerated without adverse events, despite the occasional concomitant use of ipratropium in combination with a short-acting inhaled $\beta_{2}$-agonist.

The benefits of a therapeutic regimen for patients hospitalized with an exacerbation of COPD that includes the regular once or twice daily administration of two long-acting inhaled bronchodilators (tiotropium and a LABA) with complementary mechanisms of action are likely due to the prolonged, as well as additive, improvements in airway patency from the two drugs that minimize the need for additional as-needed therapy with short-acting bronchodilators and their associated costs. The major limitations of this study are its retrospective design, the use of historical controls and the small numbers of subjects studied during each period $(n=174$ and $n=181)$. The findings need to be replicated using a controlled, prospective, multi-institutional study design (randomized by institution or subject) with larger total sample sizes before the results can be generalized to the general population of COPD patients hospitalized with an acute exacerbation not requiring intubation.

Another question raised by the study of Drescher et $\mathrm{al}^{61}$ concerns the added efficacy (as well as the safety) of ipratropium when administered on an as-needed basis on top of twice-daily therapy with tiotropium in the in-hospital treatment of COPD exacerbations. Little data are available that address this question and what data that do exist were obtained in outpatients with stable COPD on maintenance therapy with tiotropium in whom the add-on short-term responses to ipratropium were compared with those of both placebo and a short-acting $\beta_{2}$-agonist, fenoterol. ${ }^{63}$ In 
the latter study, conducted in 60 patients, the 1-hour FEV placebo-adjusted response to ipratropium vs placebo (on top of maintenance tiotropium) was $69 \mathrm{~mL}$ (95\% CI, 34-105; $P=0.0002)$ after the first dose and $47 \mathrm{~mL}$ (95\% CI, 12-83; $P=0.008)$ after the second dose. However, the corresponding placebo-adjusted responses to fenoterol were significantly $(P<0.0001)$ greater: $154 \mathrm{~mL}(95 \% \mathrm{CI}, 119-189$; $P<0.0001)$ and $208 \mathrm{~mL}(95 \% \mathrm{CI}, 173-243 ; P<0.0001)$, respectively. There was no evidence of any increase in the frequency or intensity of adverse events after the addition of either ipratropium or fenoterol in these patients on existing tiotropium therapy. Thus, while the addition of a shortacting anticholinergic on top of a long-acting anticholinergic appears to result in significant additive bronchodilation, this benefit is less than that achieved with the add-on of a shortacting $\beta_{2}$-agonist, as expected due to the known complementary action of combining two bronchodilators with different pharmacologic mechanisms. Furthermore, it is not clear that adding the combination of ipratropium and a short-acting $\beta_{2}$-agonist to an existing long-acting anticholinergic regimen would provide any advantage over the simple addition of the short-acting $\beta_{2}$-agonist alone. On the other hand, since it has been shown that long-acting bronchodilators are less effective during an acute exacerbation of COPD than in stable patients, ${ }^{60}$ the addition of short-acting bronchodilators of both classes may have different effects (both alone and in combination) in patients during an exacerbation from those evident in stable patients. Further studies are required to address these questions.

\section{Safety of tiotropium Cardiovascular events}

Doubts about the safety of tiotoprium were raised principally by a recent meta-analysis of 12 tiotropium trials by Singh et $\mathrm{al}^{64}$ who reported an elevated risk ratio (1.58 [95\% CI, 1.21-2.06]) for tiotropium vs its comparator for a composite of cardiovascular events (cardiovascular death, nonfatal myocardial infarction [MI] and nonfatal stroke), as well an increased risk ratio, separately, for cardiovascular death (1.80 [95\% CI, 1.17-2.77]) and for MI (1.53 [95\% CI, 1.05-2.23]). This meta-analysis has been criticized for relying mainly on summary (rather than patient-level) data and for failing to control for differential exposures to the study drugs. Since more placebo/comparator patients than tiotropium-treated patients discontinued these trials prematurely, the latter were at increased risk of fatal and nonfatal adverse events simply because of their longer duration of follow-up due to the fact that patients who withdrew from the studies prematurely were not followed for fatal events to the end of the pre-defined treatment period in any of the trials analyzed by Singh et al. ${ }^{64}$ In contrast to the conclusions of the latter authors, the UPLIFT trial, in which fatal events were captured in nearly all patients throughout the entire pre-defined 4-year treatment period even in those who withdrew prematurely, found a significant $13 \%$ reduction in all-cause mortality (risk ratio [RR] 0.87 [95\% CI, 0.76-0.99]) and a significantly reduced risk ratio (RR 0.78 [95\% CI, 0.65-0.94]) for the same composite fatal/nonfatal cardiovascular index for which Singh et $\mathrm{al}^{64}$ found an elevated risk, as well as a significantly reduced (rather than elevated) risk ratio (RR; 0.73 [95\% CI, 0.65-0.94]) for cardiovascular deaths specifically. ${ }^{20}$ Moreover, analysis of serious adverse events in UPLIFT showed significantly reduced risk ratios for MI (RR 0.84 [95\% CI, 0.77-0.99]) and congestive heart failure $(0.59$ [95\% CI, 0.37-0.96]) and no increased risk of stroke (0.95 [95\% CI, 0.70-1.29]), including fatal stroke (0.82 [95\% CI, 0.40-1.66]). ${ }^{20}$ Confirmatory findings from a pooled analysis of cardiovascular safety using data from 30 tiotropium double-blind, parallel-group, placebo-controlled trials of $\geq 4$ weeks duration (including UPLIFT) have recently been reported. ${ }^{65}$ These findings should dispel earlier concerns about the cardiovascular safety of tiotropium, including the risk of stoke.

\section{Other adverse events}

According to a pooled safety analysis of data from 19 clinical trials in 4435 tiotropium and 3384 placebo patients, dry mouth (RR 3.60 [2.56-5.05]) was one of the most commonly reported adverse events. ${ }^{66}$ The RR for urinary retention was elevated (10.93 [95\% CI, 1.26-94.8]), but this event was quite rare (incidence rate 0.78 per 100 -patient years in tiotropium-treated subjects). Other side effects, including constipation and visual blurring, were consistent with the known pharmacology of anticholinergic agents and occurred infrequently. Thus, it can be concluded that tiotropium is well-tolerated with a highly favorable benefit/risk ratio.

\section{Conclusions}

Exacerbations of COPD are acute events superimposed on the course of this chronic disease that are associated with increased morbidity and mortality and account for most of the economic health care burden of the disease. Consequently, the prevention and treatment of acute exacerbations are a major goal in COPD management. Several double-blind, randomized placebo-controlled trials of 3 months to 4 years duration in patients with moderate to very severe COPD have 
demonstrated the effectiveness of tiotropium in reducing both the proportion of patients experiencing at least one exacerbation and the exacerbation incidence rate and in prolonging the time to the first exacerbation. In some studies, including the 4-year UPLIFT trial, tiotropium also had a beneficial effect in reducing the risk of hospitalization for an exacerbation. The mechanism whereby tiotropium produces these benefits probably relates to its effect in producing sustained bronchodilation and reduction in hyperinflation, thus raising the threshold for experiencing symptoms during insults that might otherwise lead to an exacerbation. In the management of an acute exacerbation, an increase in short-acting bronchodilators is recommended; the potential role of regular use of long-acting bronchodilators, such as tiotropium, in addition to short-acting agents as needed, requires further study.

\section{Disclosure}

Dr Tashkin has received fees for serving on consulting/ advisory boards from Boehringer Ingelheim, AstraZeneca, Dey Laboratories and Schering-Plough; speaker fees from Boehringer Ingelheim, Pfizer, Dey Laboratories and GlaxoSmithKline; and grant support from Almirall, Boehringer Ingelheim, Dey Laboratories, GlaxoSmithKline, Pfizer, Novartis and Sepracor.

\section{References}

1. Rodriguez-Roisin R. Toward a consensus definition of COPD exacerbations. Chest. 2000;117:398S-401S.

2. Anthonisen NR, Manfreda J, Warren CP, et al. Antibiotic therapy in exacerbations of chronic obstructive pulmonary disease. Ann Intern Med. 1987;106:196-204.

3. Stevenson NJ, Walker PP, Costello RW, et al. Lung mechanics and dyspnea during exacerbations of chronic obstructive pulmonary disease. Am J Respir Crit Care Med. 2005;172:1510-1516.

4. Spencer S, Jones PW; GLOBE study group. Time course of recovery of health status following an infective exacerbation of chronic bronchitis. Thorax. 2003;58:589-593.

5. Sethi S. Etiology and management of infections in chronic obstructive pulmonary disease. Clin Pulm Med. 1999;6:327-332.

6. Sethi S, Evans N, Grant BJB, et al. New strains of bacteria and exacerbations of chronic obstructive pulmonary disease. $N$ Engl J Med. 2002;347:465-471.

7. Fujimoto k, Yasuo M, Urushibata K, et al. Airway inflammation during stable and acutely exacerbated chronic obstructive pulmonary disease. Eur Respir J. 2005;25:640-646.

8. Perera WR, Hurst JR, Wilkinson TMA, et al. Inflammaatory changes, recovery and recurrence at COPD exacerbations. Eur Respir J. 2007;29:527-534.

9. Burge S, Wedzicha JA. COPD exacerbations: definitions and classifications. Eur Respir J. 2003;21(Suppl 4):46S-53S.

10. Miravitlles M, Mayordomo C, Artes M, et al. Treatment of chronic obstructive pulmonary disease and its exacerbations in general practice. Respir Med. 1999;93:173-179.

11. Decramer M, Celli B, Kesten S, et al. Effects of tiotropium on outcomes in GOLD stage II COPD: Subgroup analysis from the UPLIFT (Understanding Potential Long-term Impacts on Function with Tiotropium) Study. Lancet. 2009;374:1171-1178.
12. O'Reilly JF, Williams AE, Holt K, et al. Defining COPD exacerbations: impact on estimation of incidence and burden in primary care. Prim Care Respir J. 2006;15:346-353.

13. Donaldson GC, Wedzicha JA. COPD exacerbations: 1: Epidemiology. Thorax. 2006;61:164-168.

14. Celli B. Roger S. Mitchell lecture: Chronic obstructive pulmonary disease, phenotypes and their clinical relevance. Proc Am Thorac Soc. 2006;3:461-465.

15. Seemungal TA, Donaldson GC, Paul EA, et al. Effect of exacerbations on quality of life in patients with chronic obstructive pulmonary disease. Am J Respir Crit Care Med. 1998;157:1418-1422.

16. Spencer S, Calverley PM, Burge PS, et al. Impact of preventing exacerbations on deterioration of health status in COPD. Eur Respir J. 2004;23:698-702.

17. O’Donnell DE, Revill SM, Web KA. Dynamic hyperinflation and exercise intolerance in chronic obstructive pulmonary disease. Am J Respir Crit Care Med. 2001;164:770-777.

18. Parker CM, Voduc N, Aaron SD, et al. Physiological changes during symptom recovery from moderate exacerbations of COPD. Eur Respir J. 2005;26:420-428.

19. O'Donnell DE, Fluge T, Gerken F, et al. Effects of tiotropium on lung hyperinflation, dyspnoea and exercise tolerance in COPD. Eur Respir J. 2004;23:832-840.

20. Tashkin DP, Celli B, Senn S, et al. A four-year trial of tiotropium in chronic obstructive pulmonary disease. $N$ Engl J Med. 2008;359: 1543-1554.

21. Kanner RE, Anthonisen NR, Connett JE. Lower respiratory illnesses promote FEV1 decline in current smokers but not ex-smokers with mild chronic obstructive pulmonary disease. Am J Respir Crit Care Med. 2001;164:358-364.

22. Donaldson GC, Seemungal TAR, Bhowmik A, et al. Relationship between exacerbation frequency and lung function decline in chronic obstructive pulmonary disease. Thorax. 2002;57:847-852.

23. American Thoracic Society/European Respiratory Society. Standards for the diagnosis and management of patients with COPD, Version 1.2. www.thoracic.org/go/copd. Accessed April 30, 2008.

24. Adams SG, Melo J, Luther M, et al. Antibiotics are associated with lower relapse rates in outpatients with acute exacerbations of COPD. Chest. 2000;117:1345-1352.

25. Murata GH, Gorby MS, Chick TW, et al. Treatment of decompensated chronic obstructive pulmonary disease in the emergency department correlation between clinical features and prognosis. Ann Emerg Med. 1991;20:125-129.

26. American Lung Association. Trends in chronic bronchitis and emphysema: morbidity and mortality. December 2007. www.lungusa.org. Accessed April 20, 2008.

27. Patil SP, Krishnan JA, Lechtzin N, et al. In-hospital mortality following acute exacerbations of chronic obstructive pulmonary disease. Arch Intern Med. 2003;163:1180-1186.

28. Seneff MG, Wagner DP, Wagner RP, et al. Hospital and 1-year survival of patients admitted to intensive care units with acute exacerbation of chronic obstructive pulmonary disease. JAMA. 1995;274:1852-1857.

29. Gunen H, Hacievliyagil SS, Kosar F, et al. Factors affecting survival of hospitalised patients with COPD. Eur Respir J. 2005;26:234-241.

30. Global Initiative for Chronic Obstructive Lung Disease (GOLD). Global strategy for the diagnosis, management, and prevention of chronic obstructive pulmonary disease. http://www.goldcopd.org/ Guidelineitem.asp?11 = 2\&12 = 1\&intId = 2003. Updated 2008.

31. Casaburi R, Mahler DA, Jones PW, et al. A long-term evaluation of once-daily inhaled tiotorpium in chronic obstructive pulmonary disease. Eur Respir J. 2002;19:217-224.

32. Beeh KM, Beier J, Buhl R, et al. Efficacy of tiotropium bromide (Spiriva) in patients with chronic obstructive pulmonary disease (COPD) of different severities. Pneumologie. 2006;60:3341-3346.

33. Brusasco V, Hodder R, Miravitlles M, et al. Health outcomes following treatment for six months with once daily tiotropium compared with twice daily salmeterol in patients with COPD. Thorax. 2003;58:399-404. 
34. Dusser D, Braveo M-L, Iacono P, et al. The effect of tiotorpium on exacerbations and airflow in patients with COPD. Eur Respir J. 2008;27:547-555.

35. Niewoehner DE, Rice K, Cote C, et al. Prevention of exacerbations of chronic obstructive pulmonary disease with tiotropium, a once-daily inhaled anticholinergic bronchodilator. Ann Intern Med. 2005;143:317-326.

36. Kesten S, Plautz M, Piquette CA, et al. Premature discontinuation of patients: a potential bias in COPD clinical trials. Eur Respir J. 2007;30:898-906.

37. Vincken W, van Noord JA, Greefhorst APM, et al. Improved health outcomes in patients with COPD during 1 year's treatment with tiotropium. Eur Respir J. 2002;19:209-216.

38. Briggs DD Jr, Covelli H, Lapidus R, et al. Improved daytime spirometric efficacy of tiotropium compared with salmeterol in patients with COPD. Pulm Pharmacol Ther. 2005;18:397-405.

39. Friedman M, Serby CW, Menjoge SS, et al. Pharmacoeconomic evalulation of a combination of ipratropium plus albuterol compared with ipratropium alone and albuterol alone in COPD. Chest. 1999;115:635-641.

40. Wedzicha JA, Calverley PM, Seemungal TA, et al. The prevention of chronic obstructive pulmonary disease exacerbations by salmeterol/ fluticasone propionate or tiotropium bromide. Am J Respir Crit Care Med. 2008;177:19-26.

41. O'Brien A, Russo-Magno P, Karki A, et al. Effects of withdrawal of inhaled steroids in men with severe irreversible airflow obstruction. Am J Respir Crit Care Med. 2001;164:365-371.

42. van der Valk P, Monninkhof E, van der Palen J, et al. Effect of discontinuation of inhaled corticosteroids in patients with chronic obstructive pulmonary disease: the COPE study. Am J Respir Crit Care Med. 2002;166:1358-1363.

43. Calverley PM, Anderson JA, Celli B, et al. Salmeterol and fluticasone propionate and survival in chronic obstructive pulmonary disease. N Engl J Med. 2007;356:775-789.

44. Drummond MB, Dasenbrook EC, Pitz MW, Murphy DJ, Fan E. Inhaled corticosteroids in patients with stable chronic obstructive pulmonary disease: a systematic review and meta-analysis. JAMA. 2008; 300:2407-2416.

45. Ernst P, Gonzalez AV, Brassard P, Suissa S. Inhaled corticosteroid use in chronic obstructive pulmonary disease and the risk of hospitalization for pneumonia. Am J Respir Crit Care Med. 2007;176:162-166.

46. Tashkin DP, Celli B, Kesten S, Lystig T, Mehra S, Decramer M. Longterm efficacy of tiotropium in relation to smoking status in the UPLIFT trial. Eur Respir J. 2010;35:287-294.

47. Aaron SD, Vandemheen KL, Fergusson D, et al. Tiotropium in combination with placebo, salmeterol or fluticasone-salmeterol for treatment of chronic obstructive pulmonary disease: randomized trial. Ann Intern Med. 2007;146:545-555.

48. Cazzola M, Tashkin DP. Combination of formoterol and tiotropium in the treatment of COPD. COPD. 2009;6:404-415.

49. Baker WL, Baker EL, Coleman CI. Phamacologic treatments for chronic obstructive pulmonary disease: A mixed-treatment comparison metaanalysis. Pharmacotherapy. 2009;29:891-905.
50. Nomura J, Hosoi T, Okuma Y, et al. The presence and function of muscarinic receptors on human T cells: the involvement in IL-2 and IL-2 receptor systems. Life Sci. 2003;72:2121-2126.

51. Koyama S, Rennard SI, Robbins RA. Acetylcholine stimulates bronchial epithelial cells to release neutrophil and monocyte chemotactic activity. Am J Physiol. 1992;262:L466-L471.

52. Powrie DJ, Wilkinson TMA, Donaldson GC, et al. Effect of tiotropium on sputum and serum inflammatory markers and exacerbations in COPD. Eur Respir J. 2007;30:472-478.

53. Tamaoki J, Chiyotani A, Tagaya E, et al. Effect of long term treatment with oxitropium bromide on airway secretions in chronic bronchitis and diffuse panbronchiolitis. Thorax. 1994;49:545-548.

54. Celli B, ZuWallack R, Wang S, et al. Improvement in resting inspiratory capacity and hyperinflation with tiotropium in COPD patients with increased static lung volumes. Chest. 2003;124:1743-1748.

55. Maltais F, Hamilton A, Marciniuk D, et al. Improvements in symptomlimited exercise performance over $8 \mathrm{hr}$ with once-daily tiotropium in patients with COPD. Chest 2005;128:1168-1178.

56. Aaron SK, Ferguson D, Marks GB, et al. Counting, analysing and reporting exacerbations of COPD in randomised controlled trials. Thorax 2008;63:122-128.

57. Suissa S, Ernst P, Vandemheen KL, et al. Methodologic issues in therapeutic trials. Eur Respir J. 2008;31:927-933.

58. Suissa S. Statistical treatment of exacerbations in therapeutic trials of chronic obstructive pulmonary disease. Am J Respir Crit Care Med. 2006;173:842-846.

59. Slymen D, Ayala G, Arredondo EM, et al. Analytic perspective: a demonstration of modeling count data with an application to physical activity. Epidemiol Perspect Innovations. 2006;3:1-9.

60. Di Marco F, Verga M, Santus P, Morelli N, Cazzola M, Centanni S. Effect of formoterol, tiotropium, and their combination in patients with acute exacerbation of chronic obstructive pulmonary disease: a pilot study. Respir Med. 2006;100:1925-1932.

61. Drescher G, Carnathan B, Imus S, Colice G. Incorporating tiotropium into a respiratory therapist-directed bronchodilator protocol for managing inpatients with COPD exacerbations decreases bronchodilator costs. Respir Care. 2008;53:1678-1684.

62. Colice GL, Carnathan B, Sung J, et al. A respiratory therapist-directed protocol for managing in-patients with asthma and COPD incorporating a long-acting bronchodilator. J Asthma. 2005;42:29-34.

63. Kerstjens HAM, Bantje TA, Luursema PB, et al. Effects of short-acting bronchodilaters added to maintenance tiotropium therapy. Chest. 2007;132:1493-1499.

64. Singh S, Loke YK, Furberg CD. Inhaled anticholinergics and risk of major adverse cardiovascular events in patients with chronic obstructive pulmonary disease. A systematic review and meta-analysis. JAMA. 2008;300:1439-1450.

65. Celli B, Decramer M, Leimer I, et al. Cardiovascular safety of tiotropium in patients with COPD. Chest. 2010;137(1):20-30.

66. Kesten S, Jara M, Wentworth C, et al. Pooled clinical trial analysis of tiotropium safety. Chest. 2006;130:1695-1703.
International Journal of COPD

\section{Publish your work in this journal}

The International Journal of COPD is an international, peer-reviewed journal of therapeutics and pharmacology focusing on concise rapid reporting of clinical studies and reviews in COPD. Special focus is given to the pathophysiological processes underlying the disease, intervention programs, patient focused education, and self management protocols.

\section{Dovepress}

This journal is indexed on PubMed Central, MedLine and CAS. The manuscript management system is completely online and includes a very quick and fair peer-review system, which is all easy to use. Visi http://www.dovepress.com/testimonials.php to read real quotes from published authors. 